\title{
Estudo da previsibilidade das medidas P-NB e 1-NB na elaboração da análise cefalométrica de Steiner*
}

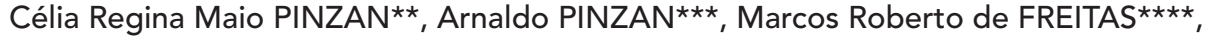

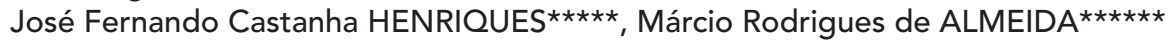

\begin{abstract}
Resumo
O propósito deste trabalho consistiu na avaliação longitudinal da previsibilidade das medidas P-NB e 1-NB na elaboração da análise de Steiner. A amostra compôs-se de 148 pacientes, divididos em três grupos de acordo com o padrão de crescimento: horizontal, equilibrado e vertical. Os valores propostos durante a individualização da análise de Steiner foram comparados com os obtidos ao final do tratamento ortodôntico e nos últimos controles realizados (em média, 4 anos e 9 meses pós-tratamento). A análise dos resultados revelou: 1) os valores propostos para a medida P-NB apresentaram-se significantemente maiores do que os obtidos ao final do tratamento nos grupos horizontal e equilibrado, e, quando comparados aos obtidos nos controles, não se verificou diferença estatisticamente significante para nenhum dos grupos avaliados; 2) os valores propostos para a medida 1-NB demonstraram diferenças estatisticamente significantes em relação aos obtidos ao final do tratamento para o grupo vertical e em relação aos obtidos nos controles, para os grupos equilibrado e vertical. Conclui-se, portanto, que se torna necessário rever as estimativas para o P-NB para pacientes com padrões de crescimento horizontal e equilibrado. Quanto à medida 1-NB, os resultados confirmaram que os valores da tabela de compromissos preconizada por Steiner subestimam o posicionamento final dos incisivos inferiores, tornando-se necessário estabelecer tabelas de compromissos aceitáveis para jovens brasileiros leucodermas com ascendência mediterrânea, de acordo com os padrões distintos de crescimento.
\end{abstract}

Palavras-chave: Cefalometria. Ortodontia corretiva.

\footnotetext{
* Resumo da dissertação apresentada à Faculdade de Odontologia de Bauru - USP, como parte dos requisitos para obtenção do título de mestre em Ortodontia.

** Doutoranda em Ortodontia pela FOB - USP; Mestre em Ortodontia pela Faculdade de Odontologia de Bauru - USP. Professora da Disciplina de Ortodontia do Departamento de Odontologia da Universidade do Sagrado Coração Bauru - SP.

*** Professor Associado do Departamento de Odontopediatria, Ortodontia e Saúde Coletiva da Faculdade de Odontologia de Bauru - USP; Professor Titular da Disciplina de Ortodontia da Universidade do Sagrado Coração - Bauru - SP; Professor da Faculdade de Odontologia da Universidade da Cidade de São Paulo - UNICID.

**** Professor Associado do Departamento de Odontopediatria, Ortodontia e Saúde Coletiva da Faculdade de Odontologia de Bauru - USP.

***** Professor Titular do Departamento de Odontopediatria, Ortodontia e Saúde Coletiva e Coordenador do Curso de Pós-Graduação em Ortodontia (Doutorado) da Faculdade de Odontologia de Bauru - USP.

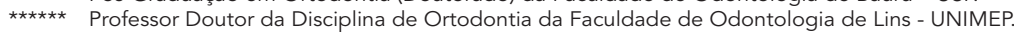




\section{INTRODUÇÃO E REVISÃO DE LITERATURA}

Com o desenvolvimento do cefalostato e a conseqüente padronização das tomadas radiográficas, em 1931, a partir dos trabalhos de Broadbent ${ }^{5}$ nos Estados Unidos e de Hofrath ${ }^{14}$ na Alemanha, ampliaram-se os conhecimentos das alterações que ocorrem no crânio, na face e nos dentes durante o crescimento e o desenvolvimento craniofacial, o que permitiu a realização de estudos radiográficos fidedignos e a proposição de inúmeras análises cefalométricas.

As análises cefalométricas fornecem valores angulares e lineares médios a partir de estudos realizados em indivíduos com oclusão normal e com perfil facial satisfatório. Esses valores propiciam comparações com os do paciente, ensejando assim verificar qual o seu padrão esquelético e dentário e quais estruturas apresentam-se discrepantes com a norma, possibilitando, por conseguinte, estabelecer o diagnóstico e o plano de tratamento setorizado e individualizado.

Historicamente, em meados da década de 40, Tweed $^{35,36}$ incluiu a movimentação dos incisivos inferiores no planejamento dos casos clínicos, com o intuito de obter uma oclusão ideal após o tratamento ortodôntico. Descreveu que estes dentes deveriam apresentar um ângulo de $90^{\circ}$ com o plano mandibular, com uma variação tolerável em torno de $+5^{0}$.

Em 1948, Downs ${ }^{7}$ determinou nove medidas angulares e uma linear que descreviam os padrões esquelético e dentário a partir de uma amostra constituída por 20 indivíduos leucodermas norte-americanos, com oclusão clinicamente excelente, desenvolvendo a primeira análise cefalométrica sistemática. Para o autor, apesar da variação considerável no tipo e no padrão facial, para que os indivíduos apresentassem um bom equilíbrio funcional e estético, deveriam possuir certas características comuns do perfil.

$\mathrm{Na}$ década seguinte, Cecil C. Steiner ${ }^{30,31,32,33}$ propôs uma análise baseada empiricamente no mecanismo compensatório dentoalveolar, orientando os profissionais a tornarem mais objetivas as elaborações dos planos de tratamento. Utilizando-se das análises de Downs, Margolis, Riedel, Thompson e Wylie combinadas com alguns valores cefalométricos de sua autoria, estabeleceu uma análise própria para jovens leucodermas de origem anglo-saxônica dos Estados Unidos.

A análise de Steiner foi bem aceita por inúmeros pesquisadores que, atentos à ocorrência de variações relacionadas aos diferentes grupos raciais ou étnicos ${ }^{26,32,33}$, propuseram-se a verificar se os valores propostos por Steiner poderiam ser aplicados às diferentes populações. Em relação, principalmente, ao padrão dentário proposto por Steiner $(1-\mathrm{NA}=4 \mathrm{~mm}$ e $1-\mathrm{NB}=$ $4 \mathrm{~mm}$ ), diversos estudos demonstraram obter valores diferentes do que os da norma propos$\operatorname{ta}^{1,9,12,13,15,16,17,18,20,24,27,28,34,37}$. Alguns autores, preocupados em melhor analisar e planejar os seus casos, estabeleceram as normas cefalométricas ideais e os compromissos aceitáveis desta análise para diferentes grupos raciais ou étnicos ${ }^{1,12,20,37}$.

Nos dias atuais, a análise proposta por Seiner ${ }^{30,31,32,33}$ ainda é mundialmente empregada durante o planejamento ortodôntico. Desta forma, propôs-se, no presente estudo, avaliar, nas fases final de tratamento ortodôntico corretivo e controle (em média 4 anos e 9 meses póstratamento), a previsibilidade das medidas $\mathrm{P}$ NB e 1-NB durante a individualização dos casos, onde se consideram as características inerentes do paciente e a mecânica a ser utilizada.

\section{PROPOSIÇÃO}

O propósito deste estudo consistiu em verificar, nas fases final de tratamento ortodôntico corretivo e controle, a previsibilidade das medidas P-NB e 1-NB estimadas na individualização da análise de Steiner, considerando-se os padrões de crescimento horizontal, equilibrado e vertical. 


\section{MATERIAL E MÉTODOS \\ Material \\ Obtenção da amostra}

A amostra utilizada para a realização deste estudo foi composta pelos valores obtidos nas telerradiografias e trabalhados no diagrama de Steiner de 148 jovens brasileiros, sendo 83 do gênero feminino e 65 do masculino, cedida gentilmente e pertencente ao acervo de documentações da Disciplina de Ortodontia, do Departamento de Odontopediatria, Ortodontia e Saúde Coletiva da Faculdade de Odontologia de Bauru - Universidade de São Paulo. Durante a seleção da amostra, os seguintes critérios foram observados:

1) pacientes portadores inicialmente de más oclusões de Classe I ou Classe II de Angle;

2) tratados no curso de pós-graduação "stricto sensu" em Ortodontia na referida instituição;

3) submetidos ou não a exodontias de pré-molares;

4) tratados ortodonticamente com aparelhos fixos, utilizando-se a técnica do arco de canto, considerando-se apenas os casos bem finalizados,

5) leucodermas, com ascendência mediterrânea (portugueses, espanhóis e italianos) e

6) suas pastas de documentações, deveriam conter as anotações referentes às medidas $\mathrm{P}-\mathrm{NB}$ e 1-NB nas fases inicial, final e último controle realizado, além da descrição dos valores propostos na individualização da análise de Steiner.

Visto que um dos critérios para se estimar os valores durante a individualização dos casos consiste na avaliação do padrão de crescimento, a amostra foi dividida, de acordo com o padrão cefalométrico de crescimento craniofacial, em três grupos:

- grupo horizontal $(\mathrm{H})$ : com 27 pacientes;
- grupo equilibrado (E): com 79 pacientes e

- grupo vertical (V): com 42 pacientes.

\section{Métodos}

Para a determinação do padrão de crescimento craniofacial, utilizou-se a medida cefalométrica SN.GoGn. Apesar da controvérsia existente na literatura em relação à confiabilidade da linha SelaNásio, selecionou-se esta medida principalmente por ser a preconizada por Steiner ${ }^{30,31,32,33}$ durante a proposição de sua análise cefalométrica.

Durante a divisão dos grupos, consultou-se o Atlas de Crescimento Craniofacial, publicado por Martins et al. ${ }^{19}$ em 1998, já que este oferece os valores médios, com os respectivos desvios padrão da medida selecionada e foi elaborado sobre uma amostra de brasileiros, descendentes de mediterrâneos (portugueses, espanhóis e italianos). Uma vez que o tratamento ortodôntico corretivo iniciase por volta dos 12 anos de idade, verificaram-se os valores para o SN.GoGn nesta idade, obtendo-se as médias de $33,2^{0}$ para o gênero feminino e de $34,3^{0}$ para o masculino. Aplicando-se os respectivos desvios padrão de $5^{0}$ para o gênero feminino e de $4,3^{0}$ para o masculino, tem-se os limites para o grupo equilibrado de $28,2^{0}-38,2^{0}$ para o feminino e de $30,3^{0}-38,6^{0}$ para o masculino. O grupo horizontal foi classificado a partir dos valores abaixo dos limites inferiores e o vertical, acima dos limites superiores. Desta forma, dividiu-se os pacientes, quanto ao padrão de crescimento de acordo com a tabela 1 .

As grandezas cefalométricas foram avaliadas nas fases: proposto, final de tratamento e controle. Verificou-se também as diferenças entre o final e o proposto $(\mathrm{F}-\mathrm{P})$ e entre o controle e proposto $(\mathrm{C}-\mathrm{P})$, sendo que para estas, o sinal negativo representa que o valor proposto foi maior que o obtido ao final do tratamento e/ou no controle. O sinal positivo indica 
que o valor proposto foi menor que o obtido ao final do tratamento e/ou no controle. Estas diferenças foram avaliadas com o intuito de observar o comportamento das estimativas, ou seja, o quanto elas foram maiores ou menores que os valores obtidos.

Para a medida P-NB, considerando-se apenas os padrões de crescimento, calculou-se as diferenças entre as fases final e inicial ( $\mathrm{F}-\mathrm{I})$, controle e final $(\mathrm{C}-\mathrm{F})$ e controle e inicial $(\mathrm{C}-\mathrm{I})$ para observar se o aumento desta medida cefalométrica apresentava-se ou não semelhante entre os grupos.

\section{ANÁLISE ESTATÍSTICA}

A estatística descritiva envolveu os cálculos das médias e dos desvios padrão (em milímetros) de cada variável cefalométrica nas fases inicial $(\mathrm{I})$, proposto $(\mathrm{P})$, final $(\mathrm{F})$, controle $(\mathrm{C})$ e para as diferença entre as fases final e proposto $(\mathrm{F}-\mathrm{P})$ e controle e proposto $(\mathrm{C}-\mathrm{P})$. Calculou-se também as médias das idades iniciais, finais, nos controles e os tempos de tratamento e do último controle realizado.

Após a obtenção desses dados, para a realização da estatística indutiva intragrupos, aplicou-se o teste " $t$ " de Student pareado com o intuito de verificar se havia ou não uma diferença estatisticamente significante entre os valores propostos e os obtidos ao final do tratamento e no último controle para cada um dos grupos estudados.

Em seguida, aplicou-se a análise de variância (ANOVA) considerando-se dois critérios: gênero e padrão de crescimento, com o intuito de verificar se os grupos e/ou os gêneros apresentavam o mesmo comportamento ou não (intergrupos). $\mathrm{Na}$ observação de um resultado estatisticamente significante $(\mathrm{p}<0,05)$, aplicou-se o teste de Tukey para indicar, especificamente, em qual(is) grupo(s) estaria(m) ocorrendo a(s) diferença(s) estatisticamente significante(s).

Novamente, utilizou-se a estatística descritiva para verificar as porcentagens de acertos e de estimativas maiores ou menores do que os valores obtidos ao final do tratamento. A fim de verificar se a porcentagem de acerto ou de estimativas maiores ou menores se distribuía uniformemente entre os padrões de crescimento horizontal, equilibrado e vertical, aplicou-se o teste do Qui-quadrado (x2), considerando-se o $\mathrm{p}<0,05$.

Considerando-se o padrão de crescimento, calculou-se apenas para o P-NB as diferenças entre as fases final e inicial ( F - I), controle e final (C - F) e controle e inicial (C - I) para verificar se a quantidade de aumento para esta medida seria ou não semelhante para os grupos horizontal, equilibrado e vertical. Aplicou-se o teste " $t$ " de Student, comparando-se os valores entre os três grupos para cada diferença avaliada a fim de observar se o aumento apresentou ou não diferença estatisticamente significante $(\mathrm{p}<0,05)$ entre os grupos.

\section{RESULTADOS E DISCUSSÃO}

As médias das idades inicial, final, nos últimos controles e as médias do tempo de tratamento e do tempo de controle, encontram-se dispostas na tabela 2, tanto separadamente, como em conjunto.

Ao analisar a tabela 2, verifica-se que os grupos apresentam as médias das idades iniciais, finais e

\begin{tabular}{|c|c|c|c|c|c|c|}
\hline & \multicolumn{6}{|c|}{$\begin{array}{l}\text { Tabela } 2 \text { - Médias das idades iniciais, finais e nos últimos controles } \\
\text { realizados e médias dos tempos de tratamento e controle, nos três grupos estudados. }\end{array}$} \\
\hline & Idade inicial & Idade final & $\begin{array}{l}\text { Idade } \\
\text { controle }\end{array}$ & $\begin{array}{c}\text { Tempo de trata- } \\
\text { mento }\end{array}$ & Tempo de controle & $\begin{array}{l}\text { Total de } \\
\text { pacientes }\end{array}$ \\
\hline $\mathrm{H}$ & 12a $11 \mathrm{~m}$ & $15 \mathrm{a} 1 \mathrm{~m}$ & 19a $9 m$ & 2a $2 m$ & $4 a 8 m$ & 27 \\
\hline$E$ & 13a $2 m$ & $15 a 3 m$ & $20 a 5 m$ & $2 a$ & $5 a 2 m$ & 79 \\
\hline V & $13 a$ & $15 a$ & $19 \mathrm{a} 6 \mathrm{~m}$ & 2a $1 \mathrm{~m}$ & $4 a 6 m$ & 42 \\
\hline $\mathrm{H}+\mathrm{E}+\mathrm{V}$ & $13 a$ & 15a $1 \mathrm{~m}$ & 19a $11 \mathrm{~m}$ & 2a $1 \mathrm{~m}$ & 4a $9 m$ & 148 \\
\hline
\end{tabular}


nos últimos controles, e dos tempos de tratamento e de controle bastante próximas entre si e em relação à amostra em geral.

\section{P-NB}

Ao descrever a sua análise, Steiner ${ }^{31}$ considerou indispensável a avaliação da distância P-NB para uma correta combinação das medidas finais. No entanto, não forneceu indicação precisa dos fatores que subsidiam seus cálculos, deixando apenas aparente o papel representado pela experiência clínica, padrão e potencial de crescimento e gênero do paciente. Afirma, em relação à previsão do P-NB que "quanto mais tem, mais terá e vice-versa".

Ao analisar a medida P-NB, em todas as fases, verifica-se, por meio da figura 1 , que houve uma tendência em se estimar maiores valores para o P-NB no grupo horizontal, um valor intermediário no grupo equilibrado e menores valores no grupo vertical. Ao final do tratamento ortodôntico, os valores obtidos no grupo vertical foram os que mais se aproximaram do valor proposto, enquanto que nos grupos horizontal e equilibrado os valores obtidos se apresentaram menores do que os propostos. Em relação aos valores obtidos nos controles, observa-se que nos três grupos estes se apresentaram maiores do que os valores estimados.

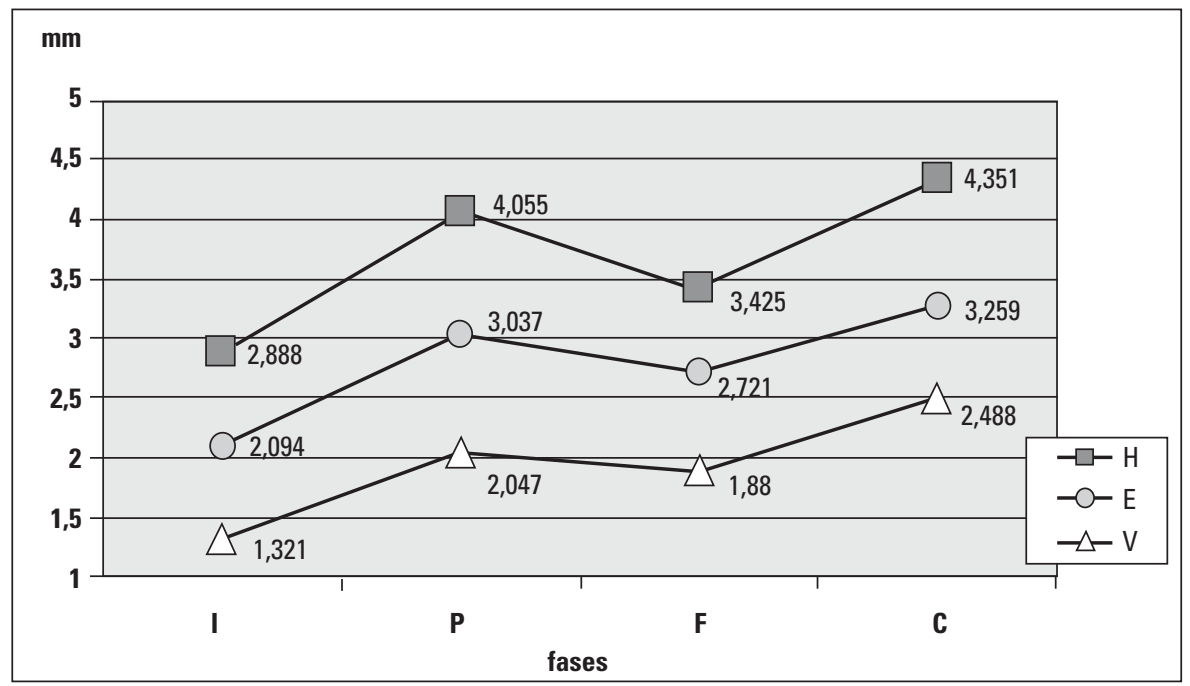

FIGURA 1- Comportamento do P-NB nas fases estudadas.

\section{P-NB (F - P)}

Nos grupos horizontal e equilibrado observou-se que os valores propostos apresentaram uma diferença estatisticamente significante dos valores obtidos ao final do tratamento (horizontal: $p=0,001$; equilibrado: $p=0,009$ ), enquanto que no grupo vertical, esta diferença não foi observada (Tab. 3).

Estas diferenças relacionam-se às estimativas maiores para o grupo horizontal, intermediárias para o equilibrado e menores para o vertical (Fig. 1), que, provavelmente, sofreram a influência da máxima de Steiner ${ }^{31,32,33}$ de que "quanto mais tem, mais terá e vice-versa".

Bishara, Jakobsen ${ }^{4}$ e Bishara ${ }^{3}$ descrevem valores maiores para o P-NB em pacientes braquifaciais e menores em dolicofaciais. Bishara ${ }^{3}$ afirma que as diferenças em relação às magnitudes dos valores do P-NB nos diferentes padrões craniofaciais encontram-se relacionadas à rotação mandibular.

Ramos et al. ${ }^{25}$, não verificaram diferença estatisticamente significante entre os valores da medida P-NB para jovens com diferentes padrões de crescimento: horizontal, equilibrado ou vertical. No entanto, ressaltaram que a ocorrência de variações individuais nesta região, pode ter mascarado a tendência do P-NB apresentar-se menor para

o grupo com padrão vertical.

Apesar da medida P-NB apresentar-se maior para o grupo com padrão horizontal e menor para o vertical nas fases inicial, final e controle, nota-se que a diferença dos valores do P-NB se manteve relativamente constante entre as fases. Não se observou um aumento significantemente maior para o grupo ho- 
rizontal e um menor, para o vertical (Tab. 4).

Quando se considerou o comportamento das estimativas entre os gêneros e/ou os padrões de crescimento, não se verificou diferença estatisticamente significante entre os grupos e/ou entre os gêneros feminino e masculino (Tab. 5).

A porcentagem de acertos durante as estimativas apresentou-se pequena (em torno de 25\%). Verificou-se um comportamento relativamente uniforme entre os diferentes padrões de crescimento nas porcentagens de acertos, estimativas maiores e menores do que os valores obtidos ao final do tratamento ortodôntico (Tab. 6). Observa-se, novamente, a tendência em estimar valores maiores do que os que foram obtidos ao final do tratamento.

\section{P-NB (C - P)}

Os resultados demonstram não ter ocorrido diferença estatisticamente significante nos grupos horizontal, equilibrado e vertical entre os valores propostos na individualização da análise de Steiner e os obtidos nos controles (Tab. 3).

Visto que os valores estimados apresentaram-se, em média, maiores do que os obtidos ao final do tratamento (Tab. 3) e ciente da ocorrência de crescimento na região anterior do mento $^{2,6,8,11,15,21,23,28,31}$ estes resultados seriam os esperados.

Não se verificou diferença estatisticamente significante no comportamento entre os grupos horizontal, equilibrado e vertical e/ou entre os gê- neros masculino e feminino em relação aos valores estimados e os obtidos nos controles (Tab. 5).

\section{1-NB}

Em 1959, Steiner ${ }^{31}$ indica a utilização da proporção de 1:1 de Holdaway, admitindo uma variação de até 3 milímetros. Desta forma, o valor estimado para o P-NB é transferido para o 1-NB do diagrama e será somado ao valor do 1-NB que se encontra na tabela de compromissos fornecida pelo autor (de acordo com o ANB estimado) e então, dividido por dois. Em seguida, este valor poderá ser individualizado. Steiner ${ }^{32}$ alerta que o valor proposto durante a individualização deve levar em consideração como e o quanto é possível alterar o posicionamento dos incisivos inferiores. Este valor obtido na individualização representa a discrepância cefalométrica da sua análise.

A discrepância cefalométrica auxilia o planejamento do caso, atuando como um indicativo da necessidade de exodontias ou de outros procedimentos, tais como a realização de desgastes proximais ou a indicação de dispositivos para a ancoragem, para melhor posicionar os incisivos inferiores.

Estudos ${ }^{18,28}$ realizados em jovens brasileiros leucodermas com oclusão normal, descendentes de mediterrâneo, demonstram que a medida 1-NB para esta população apresenta-se maior do que os valores ideais preconizados por Steiner ${ }^{30,31,32,33}$. Para a idade de 12 anos, Silva e Martins ${ }^{28}$ encontraram um valor médio para o 1-NB de 5,64 milímetros

\begin{tabular}{|c|c|c|c|c|c|c|}
\hline \multicolumn{7}{|c|}{ P-NB } \\
\hline & \multicolumn{3}{|c|}{$(F-P)$} & \multicolumn{3}{|c|}{$(C-P)$} \\
\hline & $x$ & d.p. & $\mathrm{p}$ & $x$ & d.p. & $\mathrm{p}$ \\
\hline H & $-0,63$ & 0,89 & $0,00^{*}$ & 0,30 & 1,39 & 0,28 \\
\hline E & $-0,32$ & 1,06 & $0,00^{*}$ & 0,22 & 1,34 & 0,14 \\
\hline V & $-0,17$ & 1,55 & 0,49 & 0,44 & 1,49 & 0,06 \\
\hline
\end{tabular}

* diferença estatisticamente significante $(p<0,05)$. 


\begin{tabular}{|c|c|c|c|c|c|c|}
\hline \multicolumn{7}{|c|}{ P-NB } \\
\hline & $F-I$ & $\begin{array}{l}\text { Teste "t" } \\
\mathrm{p}(\mathrm{F}-\mathrm{I})\end{array}$ & $C-F$ & $\begin{array}{l}\text { Teste " } \mathrm{t} \text { " } \\
\mathrm{p}(\mathrm{C}-\mathrm{F})\end{array}$ & C-I & $\begin{array}{l}\text { Teste " } \mathrm{t} \text { " } \\
\mathrm{p}(\mathrm{C}-\mathrm{I})\end{array}$ \\
\hline H & 0,54 & H e E: 0,69 & 0,92 & H e E: 0,07 & 1,46 & H e E: 0,21 \\
\hline$E$ & 0,63 & E e V: 0,79 & 0,42 & E e V: 0,45 & 1,04 & E e V: 0,69 \\
\hline V & 0,56 & H e V: 0,95 & 0,61 & H e V: 0,33 & 1,17 & H e V: 0,44 \\
\hline
\end{tabular}

* diferença estatisticamente signific ante $(p<0,05)$.

\begin{tabular}{|c|c|c|c|}
\hline \multicolumn{7}{c|}{ Tabela 5 - Análise de variância (ANOVA) a dois critérios - P-NB. } \\
\hline \\
\hline
\end{tabular}

* diferença estatisticamente significante $(p<0,05)$.

\begin{tabular}{|c|c|c|c|c|}
\hline \multicolumn{5}{|c|}{$\begin{array}{l}\text { P-NB } \\
(F-P)\end{array}$} \\
\hline Padrão & Acertos & Estimativas maiores & Estimativas menores & Total \\
\hline Horizontal & $22 \%(6)$ & $59 \%(16)$ & $19 \%(5)$ & $100 \%(27)$ \\
\hline Equilibrado & $25 \%(20)$ & $50 \%(39)$ & $25 \%(20)$ & $100 \%(79)$ \\
\hline Vertical & $26 \%(11)$ & $43 \%(18)$ & $31 \%(13)$ & $100 \%(42)$ \\
\hline Total & $25 \%(37)$ & $49 \%(73)$ & $26 \%(38)$ & $100 \%(148)$ \\
\hline
\end{tabular}

$x^{2}=2,004875 ; p=0,73486$

* diferença estatisticamente significante $(p<0,05)$

para o gênero masculino e de 5,46 para o feminino. Martins ${ }^{18}$ verificou uma média de 5,23 milímetros para esta medida. Uma vez que o valor considerado como ideal para Steiner ${ }^{30,31,32,33}$ é de 4 milímetros, observa-se que, para a população estudada, as normas do autor encontram-se contra-indicadas. No entanto, durante a elaboração da análise ainda utiliza-se a tabela de compromissos proposta por Steiner ${ }^{31,32,33}$.

A proporção de 1:1 de Holdaway, com um limite de tolerância de 3 milímetros foi verificada em jovens brasileiros, portadores de oclusão nor- mal e, portanto, a sua utilização durante as elaborações do diagnóstico e do plano de tratamento encontra-se indicada na literatura ${ }^{10,22}$.

No presente estudo, em relação à medida $1-\mathrm{NB}$ (Fig. 2), observa-se que nos grupos equilibrado e, principalmente, no vertical propôs-se uma retrusão dos incisivos inferiores, enquanto que no grupo horizontal estimou-se que os incisivos inferiores praticamente não sofreriam grandes alterações em relação aos seus graus de protrusão. Ao final do tratamento ortodôntico, verifica-se uma ligeira retrusão no grupo horizontal e também as retrusões 
dos incisivos inferiores tanto no grupo equilibrado como no vertical, porém em menor magnitude do que os valores propostos durante a elaboração da análise de Steiner. Nos últimos controles realizados, observa-se ainda mais uma ligeira retrusão para o grupo horizontal e ligeiras protrusões para os grupos equilibrado e vertical em relação aos valores obtidos ao final do tratamento.

Durante a avaliação desta medida, deve-se considerar o fato de que ela apresenta-se maior nos casos onde o padrão de crescimento encontrase divergente e menor nos casos com o padrão de crescimento convergente ${ }^{13,25}$. Ramos et al. ${ }^{25}$ ressaltam em seu estudo que este fato deve ser levado em consideração durante a elaboração da análise de Steiner. As médias iniciais observadas para o 1-NB demonstram esta tendência para os grupos estudados. Entretanto, observa-se que durante a proposição dos valores, esta tendência não foi considerada. Ao final do tratamento, esta diferença se manteve e nos controles, também.

\section{1-NB (F-P)}

Os valores propostos apresentaram diferenças estatisticamente significantes em relação aos valores obtidos ao final do tratamento somente no grupo vertical (Tab. 7).

Nos grupos equilibrado e, principalmente, no vertical verifica-se a proposição de uma maior retru-

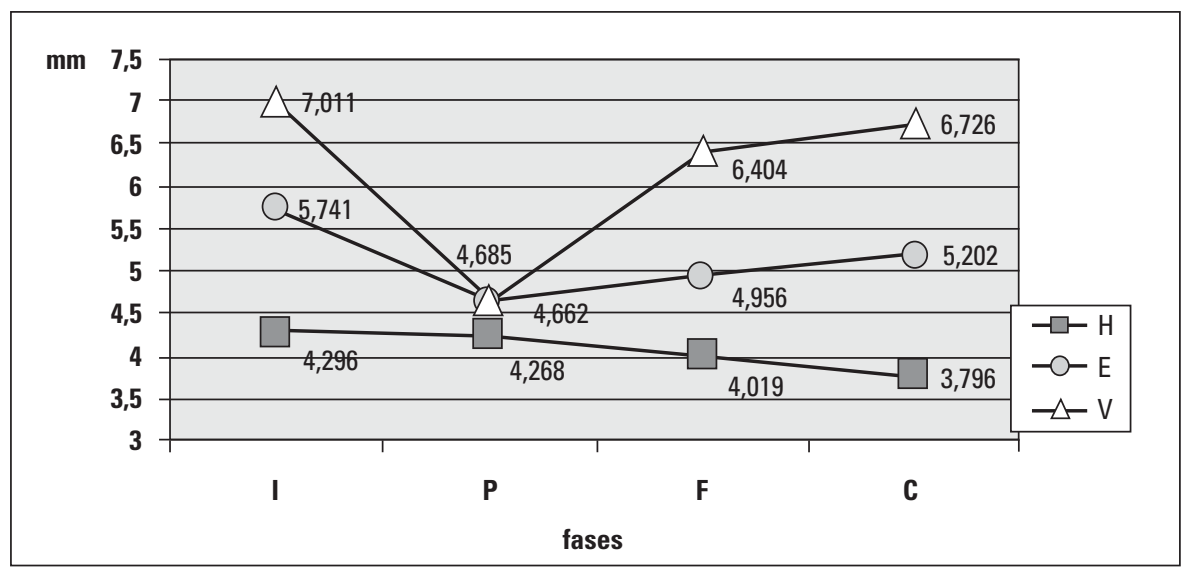

FIGURA 2- Comportamento do 1-NB nas fases estudadas. são dos incisivos inferiores do que a que foi obtida ao final do tratamento, ou seja, esta medida foi subestimada. Um comportamento semelhante foi observado por Farret e Araújo ${ }^{10}$, que também verificaram uma tendência em se subestimar os valores da medida 1-NB (médias obtidas para o 1-NB proposto: 3,62 milímetros; final: 4,98 milímetros), levando-os a concluírem que a utilização da análise de Steiner encontra-se contra-indicada para brasileiros com Classe I uma vez que os compromissos aceitáveis subestimam a posição média dos incisivos.

$\mathrm{Na}$ presente avaliação, possivelmente, não se verificou diferença estatisticamente significante entre os valores propostos e os obtidos ao final do tratamento para os grupos horizontal e equilibrado em função da utilização da proporção de 1:1 de Holdaway. Apesar dos valores fornecidos por Steiner em sua tabela de compromissos subestimarem a posição média dos incisivos inferiores para jovens brasileiros, observa-se neste estudo, que os valores estimados para a medida cefalométrica P-NB apresentaram-se significantemente maiores do que os obtidos ao final do tratamento para os grupos horizontal e equilibrado (Tab. 3). Este fato provavelmente compensou a deficiência dos valores da tabela de compromissos de Steiner, fazendo com que para estes grupos não se verificasse diferença estatisticamente significante entre os valores avaliados. Possivelmente, os valores propostos para o l-NB foram mascarados pela maior estimativa do $\mathrm{P}-\mathrm{NB}$ nos grupos com padrões horizontal e equilibrado.

Em contrapartida, no grupo vertical verificou-se uma diferença estatisticamente significante entre os valores propostos e os obtidos (Tab. 7 a 9). Observouse que estes valores foram subestimados 
Tabela 7 - Médias (X) e desvios padrão (d.p.) das diferenças entre os valores obtidos ao final do tratamento e os propostos na individualização da análise de Steiner $(\mathrm{F}-\mathrm{P})$ e das diferenças entre os valores obtidos no controle e os propostos na individualização da análise de Steiner (C - P) para a medida 1-NB e os resultados do teste " $t$ " (p).

\begin{tabular}{|c|c|c|c|c|c|c|}
\hline \multicolumn{7}{|c|}{$1-N B$} \\
\hline & \multicolumn{3}{|c|}{$F-P$} & \multicolumn{3}{|c|}{$C-P$} \\
\hline & $x$ & d.p. & Teste " $\mathrm{t}$ " $\mathrm{p}$ & $\mathrm{x}$ & d.p. & Teste " $t$ " $p$ \\
\hline H & $-0,25$ & 1,51 & 0,40 & $-0,47$ & 1,79 & 0,18 \\
\hline$E$ & 0,29 & 2,09 & 0,21 & 0,54 & 2,29 & $0,04^{*}$ \\
\hline V & 1,72 & 2,11 & $0,00^{*}$ & 2,04 & 2,14 & $0,00^{*}$ \\
\hline
\end{tabular}

* diferença estatisticamente significante $(p<0,05)$.

Tabela 8 - Análise de variância (ANOVA) a dois critérios - 1-NB .

\begin{tabular}{|c|c|c|c|c|}
\hline \multicolumn{5}{|c|}{$1-N B$} \\
\hline & & Gênero & Padrão de crescimento & Padrão de crescimento +Gênero \\
\hline$F-P$ & $\mathrm{p}$ & 0,94 & $0,00^{*}$ & 0,77 \\
\hline$C-P$ & $p$ & 0,14 & $0,00^{*}$ & 0,74 \\
\hline
\end{tabular}

* diferença estatisticamente significante $(p<0,05)$.

\begin{tabular}{|c|c|c|c|c|c|c|}
\hline \multicolumn{7}{|c|}{ Tabela 9 - Teste de Tukey. } \\
\hline \multicolumn{7}{|c|}{$1-N B$} \\
\hline & \multicolumn{3}{|c|}{$\mathbf{F}-\mathbf{P}$} & \multicolumn{3}{|c|}{ C - P } \\
\hline & Média & 1 & 2 & Média & 1 & 2 \\
\hline H & $-0,25$ & $x x x x$ & & $-0,41$ & $x x x x$ & \\
\hline E & 0,27 & $x x x x$ & & 0,44 & $x x x x$ & \\
\hline V & 1,77 & & $x x x x$ & 1,95 & & $x x x$ \\
\hline
\end{tabular}

Tabela 10 - Porcentagens de acertos e de estimativas maiores e menores do que os valores obtidos ao final do tratamento da medida cefalométrica 1-NB e o resultado do teste do Qui-quadrado (x2).

\begin{tabular}{|c|c|c|c|c|}
\hline \multicolumn{5}{|c|}{$\begin{array}{l}\text { Tabela } 10 \text { - Porcentagens de acertos e de estimativas maiores e menores do que os valores } \\
\text { obtidos ao final do tratamento da medida cefalométrica 1-NB e o resultado do teste do Qui-quadrado (x2). }\end{array}$} \\
\hline \multirow{2}{*}{\multicolumn{5}{|c|}{$\begin{array}{l}\text { 1-NB } \\
(F-P)\end{array}$}} \\
\hline & & & & \\
\hline Padrão & Acertos & Estimativas maiores & Estimativas menores & Total \\
\hline Horizontal & $18 \%(5)$ & $41 \%(11)$ & $41 \%(11)$ & $100 \%(27)$ \\
\hline Equilibrado & $10 \%(8)$ & $39 \%(31)$ & $51 \%(40)$ & $100 \%(79)$ \\
\hline Vertical & $9 \%(4)$ & $17 \%(7)$ & $74 \%(31)$ & $100 \%(42)$ \\
\hline Total & $11 \%(17)$ & $33 \%(49)$ & $56 \%(82)$ & $100 \%$ (148) \\
\hline
\end{tabular}

$x^{2}=10,17238 ; p=0,038^{*}$

* diferença estatisticamente significante $(p<0,05)$.

assim como no estudo de Farret e Araújo ${ }^{10}$. Visto que o P-NB estimado para este grupo não apresentou uma diferença significante em relação ao valor obtido e que o valor do 1-NB apresenta-se maior para jovens com padrão de crescimento vertical ${ }^{13,25}$, pode-se concordar que os valores da tabela de compromissos de Steiner subestimam a posição média dos incisivos inferiores.

Ao avaliar o comportamento das estimativas para o 1-NB considerando-se o padrão de cresci- 
mento e/ou o gênero, verificou-se uma diferença estatisticamente significante apenas quando se considerou o padrão de crescimento isoladamente (Tab. 8), confirmando novamente a tendência em subestimar essa medida no grupo vertical (Tab. 9).

A porcentagem de acertos para os valores propostos durante a individualização dos casos foi baixa, em torno de $11 \%$ (Tab. 10).

\section{1-NB (C - P)}

Ao confrontar os valores propostos durante a individualização da análise de Steiner com os obtidos nos controles, observa-se que eles apresentaram diferenças estatisticamente significantes nos grupos equilibrado e vertical (Tab. 7). Comparando-se as médias dos valores obtidos ao final do tratamento com as dos controles (Fig. 2), verifica-se que na fase pós-tratamento houve uma leve tendência dos incisivos em se movimentarem em direção às posições originais nos grupos equilibrado e vertical. Desta forma, os valores obtidos nos controles se tornam um pouco mais distantes do proposto. Este fato possivelmente explique o porquê do grupo equilibrado nesta fase, apresentar valores com diferenças estatisticamente significantes. Esta leve tendência em retornar à posição pré-tratamento também foi verificada por Silva ${ }^{29}$, Vilella e Mucha ${ }^{38}$. No grupo horizontal, os incisivos ainda apresentaram uma sutil retrusão, porém sem significado estatístico.

Quando se considerou o padrão de crescimento e/ou o gênero, observou-se, em relação ao comportamento, que apenas o grupo vertical apresentou uma diferença estatisticamente significante (Tab. 9, 10), pois os valores foram subestimados em maior magnitude.

\section{CONSIDERAÇÕES CLÍNICAS}

Observa-se que a medida P-NB, nas fases inicial, final e controle, apresenta valores maiores para o grupo horizontal, intermediários para o equilibrado e menores para o vertical (Fig. 1). Entretanto, nota-se que as diferenças entre estes valores praticamente se mantêm em todas as fases
(Fig. 1). Ao analisar as diferenças entre as fases final e inicial $(\mathrm{F}-\mathrm{I})$, controle e final $(\mathrm{C}-\mathrm{F})$ e controle e inicial ( $\mathrm{C}-\mathrm{I}$ ), observa-se que os aumentos do P-NB foram semelhantes entre os grupos, sem diferenças estatisticamente significantes (Tab. 4). Desta forma, não se evidenciou a máxima preconizada por Steiner ${ }^{31,32,33}$ de que "quanto mais tem, mais terá e vice-versa", pois apesar dos valores apresentarem-se maiores para o grupo horizontal e menores para o vertical em todas as fases, observou-se um aumento real semelhante desta medida cefalométrica entre os grupos.

Esta diferença dos valores para o P-NB entre os grupos horizontal e vertical, provavelmente, relacione-se à rotação mandibular, como descrito por Bishara e Jakobsen ${ }^{4}$ e não à quantidade de aposição óssea nesta região.

Uma vez que o aumento do P-NB apresenta-se semelhante entre os diferentes padrões de crescimento, os valores propostos para a medida P-NB nos grupos horizontal e equilibrado precisam ser revistos.

Em relação à medida $1-\mathrm{NB}$, os valores propostos provavelmente foram mascarados pela maior estimativa para o P-NB nos grupos equilibrado e, principalmente, no horizontal e pelo fato da medida 1-NB apresentar valores maiores no padrão vertical do que no horizontal e no equilibrado ${ }^{13,25}$. Uma vez que a tabela de compromissos aceitáveis de Steiner subestima o posicionamento dos incisivos inferiores para os jovens brasileiros leucodermas, ressalta-se a necessidade de se individualizar os valores para esta população.

\section{CONCLUSÕES}

A partir da metodologia empregada e dos resultados obtidos, parece-nos lícito concluírmos que:

- a medida P-NB apresentou os valores propostos significantemente maiores do que os obtidos ao final do tratamento para os grupos horizontal e equilibrado, tornando-se necessário rever os critérios para a estimativa do P-NB nesses grupos, durante a individualização no planejamento, pois somente na fase de 
controle, aqueles valores foram encontrados;

- a quantidade de aumento da medida P-NB apresentou-se semelhante entre todos os grupos, contrariando a máxima de Steiner de que "quanto mais tem, mais terá e vice-versa";

- a medida 1-NB demonstrou diferença estatisticamente significante dos valores obtidos ao final do tratamento no grupo vertical. No entanto, os resultados indicam que os valores fornecidos na tabela de compromissos aceitáveis de Steiner subestimam o posicionamento dos incisivos inferiores;

- torna-se necessário desenvolver tabelas de compromissos aceitáveis para a análise de Steiner que possam ser aplicadas à jovens brasileiros, leucodermas com descendência mediterrânea de acordo com os padrões distintos de crescimento.

\title{
Assessing the predictability of the P-NB and 1-NB values by the Steiner's cepha- lometric analysis
}

\begin{abstract}
The Steiner's cephalometric analysis has been clinically used worldwide and since its first introduction in the literature, the P-NB rationale has deemed to be essential to properly match all measures at the end of the treatment. However, the factors upon which it is estimated has so far remained uncertain and no guidelines concerning the clinical experience, growth and heredity have yet been determined. Hence, this study aims to investigate both P-NB and 1-NB predictability by the Steiner's cephalometric analysis. The sample, comprising 148 Brazilian teenagers treated at Bauru Dental School, was divided into 3 groups according to their facial growth pattern: horizontal, well balance and vertical. The P-NB and 1-NB values, attributed at the analysis individualization, were either compared to those found at the end of the treatment, and to the values achieved 4 years and 9 months posttreatment. This investigation shed the following findings: 1) The estimated P-NB values were found to be higher than those achieved after the treatment, specially for the horizontal and well balanced groups, but demonstrated to nearly equal those obtained 4 years posttreatment. 2) Only in the vertical group, the estimated l-NB values were significantly lower than those found at the end of the treatment. When comparing to posttreatment values in the long-term, differences for the well balanced and vertical groups could be attained.
\end{abstract}

Key words: Cephalometry. Orthodontic corrective. 


\section{REFERÊNCIAS}

1. ANDERSON, A. A. et al. Biological derivation of a range of cephalometric norms for children of African American descent (after Steiner). Am J Orthod Dentofacial Orthop, St. Louis, v. 118, no. 1, p. 90-100, July 2000.

2. BAUM, A. T. Age and sex differences in the dentofacial changes following orthodontic treatment and their significance in treatment planning. Am J Orthod, St. Louis, v. 47, no. 5, p. 355-370, May 1961.

3. BISHARA, S. E. Facial and dental changes in adolescents and their clinical implications. Angle Orthod, Appleton, v. 70, no. 6, p. 471-483, Dec. 2000.

4. BISHARA, S. E.; JAKOBSEN, J. R. Longitudinal changes in three normal facial types. Am J Orthod, St. Louis, v. 88, no. 6, p. 466-502, Dec. 1985.

5. BROADBENT, B. H. A new X-ray technique and its application to orthodontia. Angle Orthod, Appleton, v. 1, no. 2, p. 45-66 Apr. 1931

6. COURA, L.C.; PINZAN, A.; FREITAS, M. R. Estudo cefalométrico longitudinal do complexo mandibular em pacientes adultos do sexo masculino tratados ortodonticamente com extração de quatro pré-molares. Ortodontia, São Paulo, v. 30, n. 1, p.1930, jan./abr. 1997.

7. DOWNS, W. B. Variations in facial relationships: their significance in treatment and prognosis. Am J Orthod, St. Louis, v. 34, no. 10, p. 812-840, Oct. 1948.

8. DRUMMOND, A.F. Estudo das alterações esqueléticas das fases final, cinco e dez anos pós-tratamento, em pacientes do sexo feminino, tratados ortodonticamente, pela técnica do arco de canto simplificada. 1991. 88 f. Dissertação (Mestrado)-Faculdade de Odontologia de Bauru, Universidade de São Paulo, Bauru, 1991.

9. EVANKO, A. M.; FREEMAN, K.; CISNEROS, G. J. Mesh diagram analysis: developing a norm for Puerto Rican Americans. Angle Orthod, Appleton, v. 67, no. 5, p. 381-388, Oct. 1997.

10. FARRET, M. M. B.; ARAÚJO, M. C. M. Comportamento da análise de Steiner em casos tratados ortodonticamente. Ortodontia, São Paulo, v. 14, n. 3, p.164-172, set./dez. 1981.

11. FÊO, P. S. Aumento da distância Pog-NB na adolescência. Estomatol Cult, Baurú, v. 4, n. 1, p. 5-14, jan./jun. 1970.

12. GLEIS, R.; BREZNIAK, N.; LIEBERMAN, M. Israeli cephalometric standards compared to Downs and Steiner analyses. Angle Orthod, Appleton, v. 60, no. 1, p. 35-40, Spring 1990

13. HASUND, A.; ULSTEIN, G. The position of the incisors in relation to the lines NA and NB in different facial types. Am J Orthod, St. Louis, v. 57, no. 1, p.1-14, Jan.1970.

14. HOFRATH, H. Problems of standard and methods. In: KROGMAN, W. M.; SASSOUNI, V. A sillabus in roentgenographic cephalometry. 2nd ed. Philadelphia: College Offset, 1957. cap. 1, p. 9-25.

15. HOPKINS, J.B.; MURPHY, J. Variations in good occlusions. Angle Orthod, Appleton, v. 41, no. 1, p. 55-65, Jan. 1971

16. KOWALSKI, C. J.; WALKER, G. F. The use of incisal angles in the Steiner cephalometric analysis. Angle Orthod, Appleton, v. 42, no. 2, p. 87-95, Apr. 1972.

17. KOWALSKI, C. J.; NASJLETI, C. E.; WALKER, G. F. Differential diagnosis of adult male black and white populations. Angle Orthod, Appleton, v. 44, no. 4, p. 346-350, Oct. 1974.

18. MARTINS, D.R. Estudo comparativo dos valores cefalométricos das análises de Downs, Tweed, Steiner e Alabama, com os de adolescentes brasileiros, leucodermas, de origem mediterrânea. 1979. 141 f. Tese (Livre docência)-Faculdade de Odontologia de Bauru, Universidade de São Paulo, Bauru, 1979

19. MARTINS, D. R. et al. Atlas de crescimento craniofacial. São Paulo: Ed. Santos, 1998.
20. MIURA, F.; INOVE, N.; SUZUKI, K. Cephalometric standards for japanese according to the Steiner analysis. Am J Orthod, St. Louis, v. 51, no. 4, p. 288-295, Apr. 1965.

21. NANDA, R. S. Growth changes in skeletal-facial profile and their significance in orthodontic diagnosis. Am J Orthod, St. Louis, v. 59, no. 5, p. 501-513, May 1971

22. OLIVEIRA, J. N.; MARTINS, D.R. Estudo longitudinal e comparativo da variação do pogônio com os incisivos inferiores, em relação à linha NB, em adolescentes brasileiros, leucoderma, de 12 aos 18 anos de idade, com "oclusão normal". Ortodontia, São Paulo, v. 11, n. 2, p. 9 9-107, maio/ago. 1978.

23. PINZAN, A. Estudo comparativo da medida P-NB, em adolescentes leucodermas, brasileiras, de origem mediterrânea, com normal e más oclusões (Classe I e Classe II, divisão 1), tratadas ortodonticamente. Ortodontia, São Paulo, v. 20, n. 1/2, p. 47-51, jan./dez. 1987.

24. PLATOU, C.; ZACHRISSON, B. Incisor position in Scandinavian children with ideal occlusion: a comparison with the Ricketts and Steiner standards. Am J Orthod, St. Louis, v.83, no. 4, p.341-351, Apr. 1983.

25. RAMOS, A. L. et al. Influência da divergência facial no posicionamento dentário e das bases apicais, em jovens brasileiros com oclusão normal. Ortodontia, São Paulo,v. 29, n. 3 p. 44-54, set./dez. 1996.

26. SALZMANN, J. A. The research workshop on cephalometrics. Am J Orthod, St. Louis, v. 46, no. 11, p. 834 847, Nov. 1960.

27. SERVOSS, J. M. The acceptability of Steiner's acceptable compromises. Am J Orthod, St. Louis, v. 63, no. 2, p. 161-165, Feb. 1973.

28. SILVA, L. G.; MARTINS, D. R. Determinação dos valores cefalométricos 1.NA, 1.NB, 1-NA e 1-NB para adolescentes brasileiros, leucodermas, com "oclusão normal", (estudo longitudinal e comparativo). Ortodontia, São Paulo, v. 11, n. 2, p. 108-116, maio/ago. 1978

29. SILVA, M. L. A. Estudo cefalométrico semilongitudinal (fases inicial, final de tratamento e pós-contenção) de jovens brasileiras, leucodermas, tratadas ortodonticamente, portadoras inicialmente de Classes I e II divisão 1 e comparadas com as de oclusão normal. 1986. 110 f. Dissertação (Mestrado)-Faculdade de Odontologia de Bauru, Universidade de São Paulo, Bauru, 1986.

30. STEINER, C. C. Cephalometrics for you and me. Am J Orthod St. Louis, v. 39, no. 10, p. 729-755, Oct. 1953.

31. STEINER, C. C. Cephalometrics in clinical practice. Angle Orthod, Appleton, v. 29, no. 1, p. 8-29, Jan. 1959.

32. STEINER, C. C. The use of cephalometrics as an aid to planning and assessing orthodontic treatment. Am J Orthod, St. Louis, v. 46, no. 10, p. 721-735, Oct. 1960.

33. STEINER, C. C. Cephalometrics as a clinical tool. In: KRAUS, B. S.; RIEDEL, R. A. Vistas in orthodontics. Philadelphia: Lea \& Febiger, 1962. p. 131-161.

34. TAYLOR, W. H.; HITCHCOCK, H. P. The Alabama analysis. Am J Orthod, St. Louis, v. 52, no. 4, p.245-265, Apr. 1966.

35. TWEED, C. H. A philosophy of orthodontic treatment. Am J Orthod Oral Surg, St. Louis, v. 31, no. 2, p. 74-103, Feb. 1945.

36. TWEED, C. H. The Frankfort: mandibular plane angle in orthodontic diagnosis, classification, treatment planning and prognosis. Am J Orthod Oral Surg, St. Louis, v. 32, no. 4, p.175-221, Apr. 1946.

37. UESATO, G. et al. Steiner cepphalometric norms for Japaneses and Japanese-Americans. Am J Orthod, St. Louis, v. 73, no. 3 p.321-327, Mar. 1978.

38. VILELLA, O. V.; MUCHA, J. N. Avaliação cefalométrica da estabilidade dos incisivos inferiores pós-contenção ortodôntica. Ortodontia, São Paulo, v. 29, n. 1, p. 48-52, jan./abr. 1996. 